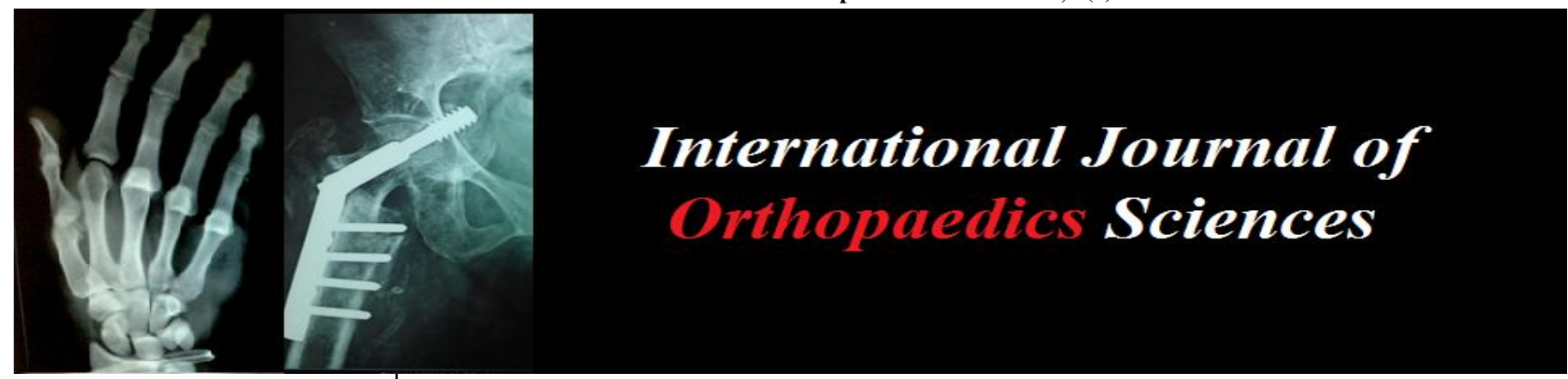

E-ISSN: 2395-1958

P-ISSN: 2706-6630

IJOS 2020; 6(2): 728-733

(C) $2020 \mathrm{IJOS}$

www.orthopaper.com

Received: 18-02-2020

Accepted: 19-03-2020

Dr. Shyam Ramesh

Post graduate resident,

Department of Orthopaedics,

KVG Medical College, Sullia,

Karnataka, India

Dr. Roshan SD

Associate Professor, Department of Orthopaedics, KVG Medical

College, Sullia, Karnataka, India

Dr. Chennakeshava Rao

Professor and HOD, Department

of Orthopaedics, KVG Medical

College, Sullia, Karnataka, India
Corresponding Author:

Dr. Roshan SD

Associate Professor, Department of Orthopaedics, KVG Medical

College, Sullia, Karnataka, India

\section{Nonoperative treatment compared with volar locking plate fixation for displaced and unstable distal radius fractures in patients sixty-five years of age and older - A prospective randomized trial}

\author{
Dr. Shyam Ramesh, Dr. Roshan SD and Dr. Chennakeshava Rao
}

DOI: https://doi.org/10.22271/ortho.2020.v6.i21.2129

\begin{abstract}
Recent trends have shown a decrease in nonoperative management for unstable distal radial fractures in elderly. The literatures available lack adequate information whether operative treatments are superior to nonoperative (cast) treatment. The purpose of this study was to compare the outcomes of two treatment methods that were used: (1) ORIF with volar locking plate and (2) closed reduction and plaster immobilization. 70 patients with a displaced and unstable distal radial fracture were randomized to ORIF with volar locking plate $(n=35)$ or closed reduction and cast immobilization $(n=35)$. Outcome was measured on the basis of the Patient-Rated Wrist Evaluation (PRWE) score; the Disabilities of the Arm, Shoulder and Hand (DASH) score; the pain level; the range of wrist motion; the rate of complications; and radiographic measurements including dorsal radial tilt, radial inclination, and ulnar variance. Patients in the operative treatment group had lower DASH and PRWE scores, indicating better wrist function in early postoperative period $(p<0.05)$, but there were no significant differences between the groups at six and twelve months. Grip strength, dorsal radial tilt, radial inclination, and radial shortening were significantly better in the operative treatment group at the time of the latest follow-up $(p<0.05)$
\end{abstract}

Keywords: Volar Locking Plate Fixation, Displaced and Unstable Distal Radius Fractures, A Prospective Randomized Trial

\section{Introduction}

Distal radius fractures are the most common fractures encountered in health care [1]. Considering the increasing demands of today's elderly population with increasing life expectancy, the appropriate treatment of these fractures is of growing importance. The current literature on the treatment of distal radial fractures in the elderly is more controversial. Stable fractures can be treated with closed reduction and cast immobilization, with satisfactory outcomes ${ }^{[2]}$. Results from a randomized controlled study published in 2018 revealed that, in comparison with non-operative treatment, significantly better function was recorded 2 years after the surgical procedure for patients treated with a volar plate ${ }^{[3]}$. Some authors have even suggested that elderly patients with unstable distal radial fractures should be managed nonoperatively because fracture reduction and anatomic alignment on radiographs are not correlated with better functional outcomes in older patients as compared with younger patients ${ }^{[4-7]}$. The usage of volar locking plates has increased in older populations ${ }^{[8]}$, although surgical treatment of unstable fractures among the elderly population has until recently not been proven to be superior to nonoperative treatment with regard to clinical outcome ${ }^{[9-13]}$. The aim of this randomized clinical trial was to compare the outcomes of two methods for the treatment of displaced and unstable distal radial fractures in patients sixty-five years of age or older: (1) open reduction and internal fixation (ORIF) with use of a volar fixed-angle implant and (2) closed reduction and plaster immobilization (casting)

\section{Methodology}

The trial was conducted at the Department of Orthopaedic Surgery, KVG Medical college, Sullia, Karnataka, India. Institutional approval was obtained from the ethical committee prior 
to the initiation of the study. All patients provided written informed consent in their own understandable language, before their participation in the study. Eligible patients (as described below) with unstable dorsally displaced distal radial fractures were randomized to operative or nonoperative treatment. The primary outcome measure was the PatientRated Wrist Evaluation (PRWE) score [14], and secondary outcome measures included the Disabilities of the Arm, Shoulder and Hand (DASH) score ${ }^{[15]}$, the level of pain, the range of wrist motion, and the rate of complications. Our hypothesis was that there would be no difference between the operative and nonoperative treatment groups with respect to the PRWE score and objective surgeon-based functional measurements. From 2018 to 2019, 253 patients with an age of sixty-five years or more who had a distal radial fracture were managed at our institution and were evaluated for eligibility. Of these, 162 patients did not match the inclusion criteria. Eleven patients refused randomization, insisting on nonoperative treatment, and were excluded from the study. Remaining eighty patients (eighty fractures) met the inclusion criteria, agreed to participate, and were entered in the study. The mechanism of injury was a fall from a standing height in all cases. Forty patients were randomized to ORIF (the operative treatment group), and forty patients were randomized to casting (the nonoperative treatment group). Two patients in the operative treatment group and three patients in the nonoperative treatment group died of unrelated conditions before the latest follow-up examination and were excluded. Three patients from the operative treatment group and two patients from the nonoperative treatment group were lost to follow-up and were excluded. The remaining seventy patients (eighteen men and fifty-two women) with a mean age of 79.4 years of age (sixty-five to ninety years old) completed the study. Thus, thirty-five patients in the operative treatment group and thirty-five patients in the non-operative treatment group were available for the one year follow-up examination. In order to be included in the present study, a patient had to meet one or more of the inclusion criteria. Primary reduction of the fracture was considered to be acceptable when the dorsal radial tilt did not exceed $10^{\circ}$, radial shortening was not more than $2 \mathrm{~mm}$, and any intraarticular step-off did not exceed $1 \mathrm{~mm}^{[4]}$.

Patients with stable fractures were excluded from the study.

\section{Sample Size Calculation}

Before the study, a sample size analysis was performed. The sample size was chosen on the basis of the primary outcome of wrist function and the wrist scores utilized. We assumed an alpha error of 0.05 and applied an allocation ratio of 1 . A sample size of sixty-eight participants was calculated to provide $80 \%$ power to detect a difference of 10 points in the PRWE score, which we consider to be clinically relevant.

\section{Randomization}

Initially, all fractures were reduced with the patient under hematoma block and were fixed with a forearm plaster cast either in the OPD, or in the emergency department. Fracture reduction was performed either by an orthopaedic surgery resident or by an orthopaedic surgeon. Radiographs were used to assess the fracture alignment after manipulation. All patients were evaluated at one week after reduction in the outpatient department. At that time, fracture reduction in all patients was reassessed with use of radiographs. Patients with initial inadequate reduction and patients with subsequent loss of reduction at the one-week follow-up examination who were identified as being eligible for the study by an orthopaedic surgeon, were introduced to the study protocol at the oneweek follow-up examination. Patients were explained about the workflow of the study. After obtaining patient consent, the patients were randomized to either ORIF or nonoperative treatment with use of sequentially numbered, sealed envelopes.

\section{Operative Treatment}

Patients who were randomized to the ORIF group had operative treatment within fourteen days after the injury. In thirty-two patients, surgery was performed with use of a brachial plexus block and in three patients surgery wasperformed with use of general anesthesia and an upper arm tourniquet. The distal part of the radius was exposed through a palmar approach along the flexor

carpi radialis tendon. After the release of the pronator quadratus muscle, the fracture site was exposed. Fracture reduction was achieved with the assistance of an image intensifier, and the fracture site was temporarily stabilized with Kirschner wires. The volar fixed-angle plate (2.4-mm LCP DRP) was placed on the volar radial cortex and was first fixed at the gliding hole to allow appropriate positioning for the image-controlled subchondral placement of the interlocking screws. If possible, the pronator quadratus muscle was reinserted to protect the flexor tendons. All surgical procedures were performed by experienced orthopaedic surgeons who determined the choice of implant. No additional bone grafting was used. After surgery, the wrist was immobilized in a below-the-elbow splint for pain reduction. The active digital range of motion was started immediately. Ten days after surgery, the sutures were removed and the wrist was placed in a removable splint for another week. At that time, physiotherapy with active and passive wrist mobilization out of the splint was started.

\section{Nonoperative Treatment}

No repeated manipulation was done for fractures that were treated nonoperatively. We believe that elderly patients with unstable distal radial fractures do not benefit from remanipulation as the incidence of complex regional pain syndrome (CRPS) Type I is higher in such patients ${ }^{16}$. All wrists were immobilized in a short arm cast in a neutral position for five weeks. The active digital range of motion was started immediately. After cast removal, physiotherapy was started.

\section{Outcome}

All patients were assessed clinically and radiographically at six and twelve weeks and at six and twelve months after the fracture.

\section{Functional Assessment}

At each visit, a clinical examination was performed by an independent examiner who was not blinded to the method of treatment because the palmar scars in the operative treatment group could be easily observed. Functional assessment included the measurement of active wrist extension, flexion, pronation, and supination with use of a goniometer. Grip power was measured with a dynamometer. All clinical values for the involved side were compared with those for the contralateral side. Wrist pain was evaluated with use of a visual analog scale (VAS) (with 0 representing no pain and 10 representing severe pain). The functional subjective outcome was measured with the DASH questionnaire (range, 0 to 100 , 
with 0 as the best result) and the PRWE score (range, 0 to 100, with 0 indicating that the patient was asymptomatic). The presence of an obvious clinical wrist deformity was recorded, and patients were asked whether the appearance of the wrist was bothersome. Patients were also asked with use of a simple yes-or-no question if they were satisfied with the end result of the treatment. An adverse event was defined as any event that necessitated another surgical intervention or additional medical treatment. Complications included loss of reduction, fracture malunion, and fracture nonunion as well as deep infection, neuropathy and tendon irritation. Complex regional pain syndrome was diagnosed on the basis of the presence of dysaesthetic pain and hyperesthesia extending into the hand of the injured limb, vasomotor changes, skin atrophy, and diffuse osteopenia ${ }^{[17]}$.

\section{Radiographic Assessment}

Standard anteroposterior and lateral radiographs were made and were evaluated with regard to radial dorsal tilt, radial inclination, ulnar variance, and intra-articular step-off immediately after surgery for the operative treatment group and immediately after closed reduction for the nonoperative treatment group. Follow-up radiographs were made at one week, six weeks, and twelve weeks and at six and twelve months for both groups. Fracture union was defined as bone bridging of the radial, ulnar, and dorsal cortical aspects of the distal part of the radius as seen on antero-posterior and lateral projections. Post-traumatic arthritis was classified with use of the system of Knirk and Jupiter ${ }^{[18]}$. The assessor of the radiographic outcome was not a treating surgeon and was blinded to the functional outcome but not to the method of treatment.

\section{Statistical Analysis}

IBM SPSS version 17 software was used for statistical analysis. For numerical variables, normality was tested with the Shapiro-Wilk test. A nonparametric Mann-Whitney U test was performed for the determination of differences of mean values between the two treatment groups. In the metaanalyses, the mean differences are presented with a 95\% confidence interval (CI). The chi-square test or Fisher exact test was used for categorical variables; tests were two sided and presented with $\mathrm{p}$ values, and the level of alpha was set at $p<0.05$

Results:

Table 1: Clinical outcomes

\begin{tabular}{|c|c|c|c|}
\hline & Operative treatment group & Nonoperative treatment group & P Value \\
\hline \multicolumn{4}{|c|}{6 weeks } \\
\hline Extension* (deg) & $46 \pm 15(76.1 \%)$ & $45 \pm 15(75.4 \%)$ & 0.67 \\
\hline Flexion* $(\mathrm{deg})$ & $44 \pm 13(72.3 \%)$ & $41 \pm 11(72.7 \%)$ & 0.42 \\
\hline Supination* (deg) & $75 \pm 18(88.6 \%)$ & $73 \pm 16(85.4 \%)$ & 0.61 \\
\hline Pronation* $(\mathrm{deg})$ & $81 \pm 9(96.0 \%)$ & $77 \pm 16(91.3 \%)$ & 0.21 \\
\hline Ulnar deviation*(deg) & $26 \pm 8(76.3 \%)$ & $26 \pm 11(73.0 \%)$ & 0.78 \\
\hline Radial deviation*(deg) & $18 \pm 8(84.1 \%)$ & $19 \pm 8(81.4 \%)$ & 0.86 \\
\hline Grip strength* $(\mathrm{kg})$ & $14.1 \pm 5.5(60.3 \%)$ & $10.7 \pm 5.6(54.9 \%)$ & 0.01 \\
\hline Pain at rest $\uparrow$ (points) & $0.3 \pm 0.8$ & $0.3 \pm 0.8$ & 0.97 \\
\hline Pain under stress $\dagger$ (points) & $2.0 \pm 2.0$ & $2.5 \pm 2.1$ & 0.24 \\
\hline DASH score $†$ (points) & $18.8 \pm 17.9$ & $34.4 \pm 22.5$ & 0.00 \\
\hline PRWE score $\uparrow$ (points) & $36.4 \pm 28.7$ & $64.9 \pm 29.0$ & 0.00 \\
\hline \multicolumn{4}{|c|}{12 weeks } \\
\hline Extension* (deg) & $51 \pm 13(85.6 \%)$ & $52 \pm 9(87.4 \%)$ & 0.88 \\
\hline Flexion* $(\mathrm{deg})$ & $47 \pm 12(80.0 \%)$ & $49 \pm 11(81.4 \%)$ & 0.49 \\
\hline Supination* (deg) & $80 \pm 14(93.4 \%)$ & $80 \pm 12(95.7 \%)$ & 0.96 \\
\hline Pronation* $(\mathrm{deg})$ & $81 \pm 13(94.8 \%)$ & $81 \pm 12(96.5 \%)$ & 0.81 \\
\hline Ulnar deviation* (deg) & $30 \pm 10(91.7 \%)$ & $28 \pm 10(79.5 \%)$ & 0.38 \\
\hline Radial deviation* (deg) & $20 \pm 7(82.2 \%)$ & $20 \pm 7(89.0 \%)$ & 0.94 \\
\hline Grip strength* $(\mathrm{kg})$ & $15.7 \pm 6.2(77.1 \%)$ & $12.5 \pm 4.4(58.3 \%)$ & 0.02 \\
\hline Pain at rest $\uparrow$ (points) & $0.2 \pm 0.7$ & $0.3 \pm 0.8$ & 0.78 \\
\hline Pain under stress $\dagger$ (points) & $1.4 \pm 2.0$ & $1.8 \pm 2.0$ & 0.40 \\
\hline DASH score $\uparrow$ (points) & $13.3 \pm 14.8$ & $23.2 \pm 19.3$ & 0.02 \\
\hline PRWE score $\uparrow$ (points) & $33.7 \pm 32.0$ & $54.4 \pm 31.8$ & 0.01 \\
\hline \multicolumn{4}{|c|}{6 months } \\
\hline Extension* (deg) $(91.1 \%)$ & $55 \pm 11$ & $55 \pm 12(94.6 \%)$ & 0.99 \\
\hline Flexion* (deg) $(88.4 \%)$ & $51 \pm 10$ & $48 \pm 13(83.1 \%)$ & 0.28 \\
\hline Supination* (deg) $(97.1 \%)$ & $83 \pm 9$ & $79 \pm 12(93.7 \%)$ & 0.13 \\
\hline Pronation* (deg) $(99.3 \%)$ & $84 \pm 8$ & $81 \pm 14(96.3 \%)$ & 0.20 \\
\hline Ulnar deviation* (deg) & $33 \pm 9(98.3 \%)$ & $30 \pm 10(88.8 \%)$ & 0.20 \\
\hline Radial deviation* (deg) & $20 \pm 7(83.8 \%)$ & $21 \pm 12(109.2 \%)$ & 0.51 \\
\hline Grip strength* (kg) (88.4\%) & $19.8 \pm 7.4$ & $16.1 \pm 5.6(79.0 \%)$ & 0.02 \\
\hline Pain at rest $\dagger$ (points) & $0.6 \pm 1.3$ & $0.3 \pm 1.0$ & 0.30 \\
\hline Pain under stress $\dagger$ (points) & $1.3 \pm 1.7$ & $1.0 \pm 1.6$ & 0.43 \\
\hline DASH score $\dagger$ (points) & $12.2 \pm 14.4$ & $12.4 \pm 17.0$ & 0.94 \\
\hline PRWE score $\dagger$ (points) & $27.7 \pm 32.0$ & $31.4 \pm 33.0$ & 0.63 \\
\hline \multicolumn{4}{|c|}{12 months } \\
\hline Extension* (deg) & $59 \pm 10(94.4 \%)$ & $61 \pm 7(106.6 \%)$ & 0.14 \\
\hline Flexion* (deg) & $55 \pm 11(91.1 \%)$ & $57 \pm 10(100.7 \%)$ & 0.50 \\
\hline Supination* (deg) & $85 \pm 8(99.6 \%)$ & $85 \pm 8(100.4 \%)$ & 0.99 \\
\hline Pronation* (deg) $(99.4 \%)$ & $84 \pm 7$ & $85 \pm 8(100.8 \%)$ & 0.53 \\
\hline
\end{tabular}




\begin{tabular}{|c|c|c|c|}
\hline Ulnar deviation* (deg) & $35 \pm 8(101.6 \%)$ & $35 \pm 8(102.4 \%)$ & 0.88 \\
\hline Radial deviation* $(\mathrm{deg})$ & $24 \pm 6(98.0 \%)$ & $25 \pm 7(114.9 \%)$ & 0.52 \\
\hline Grip strength* (kg) $(102.4 \%)$ & $22.2 \pm 6.3$ & $18.8 \pm 5.8(92.6 \%)$ & 0.02 \\
\hline Pain at rest $\dagger$ (points) & $0.1 \pm 0.3$ & $0.1 \pm 0.5$ & 0.80 \\
\hline Pain under stress $\dagger$ (points) & $0.7 \pm 1.0$ & $0.6 \pm 1.4$ & 0.80 \\
\hline DASH score† (points) & $5.7 \pm 11.1$ & $14.0 \pm 9.3$ & 0.34 \\
\hline PRWE score† (points) & $12.8 \pm 23.2$ & $14.6 \pm 22.8$ & 0.73 \\
\hline
\end{tabular}

*The values are given as the mean and the standard deviation, with the percentage of the value for the uninjured side in parentheses. $\dagger$ The values are given as the mean and the standard deviation.

Table 2: Radiographic Outcomes*

\begin{tabular}{|c|c|c|c|}
\hline & Operative treatment group & Nonoperative treatment group & P Value \\
\hline \multicolumn{4}{|c|}{ Palmar tilt (deg) } \\
\hline Postoperative/after closed reduction & $3.6 \pm 6.9$ & $-3.6 \pm 11.2$ & 0.00 \\
\hline Latest follow-up examination & $3.0 \pm 7.2$ & $-10.4 \pm 19.1$ & 0.00 \\
\hline Loss of reduction & $0.5 \pm 4.7$ & $6.9 \pm 17.6$ & 0.04 \\
\hline \multicolumn{4}{|c|}{ Radial inclination (deg) } \\
\hline Postoperative/after closed reduction & $21.8 \pm 2.7$ & $20.3 \pm 3.3$ & 0.04 \\
\hline Latest follow-up examination & $21.2 \pm 2.6$ & $15.9 \pm 9.0$ & 0.00 \\
\hline Loss of reduction & $0.5 \pm 2.5$ & $4.4 \pm 7.9$ & 0.01 \\
\hline \multicolumn{4}{|c|}{ Ulnar variance $(\mathrm{mm})$} \\
\hline Postoperative/after closed reduction & $0.6 \pm 1.6$ & $0.8 \pm 1.7$ & 0.57 \\
\hline Latest follow-up examination & $0.7 \pm 1.8$ & $3.2 \pm 2.9$ & 0.00 \\
\hline Loss of reduction & $-0.1 \pm 1.5$ & $-2.4 \pm 2.6$ & 0.00 \\
\hline \multicolumn{4}{|c|}{ Step-off $(\mathbf{m m})$} \\
\hline Pre-reduction & $0.5 \pm 1.1$ & $0.4 \pm 1.1$ & 0.79 \\
\hline Post-reduction & $0.2 \pm 0.6$ & $0.7 \pm 1.2$ & 0.02 \\
\hline Latest follow-up examination & $0.2 \pm 0.5$ & $0.6 \pm 1.1$ & 0.04 \\
\hline
\end{tabular}

Satisfactory primary closed reduction (defined as $<10^{\circ}$ of dorsal radial tilt, $<2 \mathrm{~mm}$ of radial shortening, and $<2 \mathrm{~mm}$ of articular incongruity) ${ }^{[19]}$ was achieved in 29 patients $(41 \%)$, incongruity occurred in all patients (100\%) in the nonoperative treatment group. At the time of the latest follow-up examination, dorsal tilt, radial inclination, radial shortening (ulnar variance), and intra-articular step-off were significantly better, and loss of reduction was significantly lower, in the operative treatment group as compared with the nonoperative treatment group ( $p<0.05 ; \mathrm{t}$ test for independent samples).

After six weeks, radiographic union was observed in thirtyfour patients $(97 \%)$ in the operative treatment group and in twenty-nine patients $(83 \%)$ in the nonoperative treatment group. The higher prevalence of delayed union in the nonoperative treatment group was not significant $(p>0.05 ; \mathrm{t}$ test for independent samples). After twelve weeks, union was observed in all patients in the operative treatment group and in thirty-three patients $(94 \%)$ in the non-operative treatment group. All fractures healed within six months.

In the operative treatment group, nine patients (35\%) with intra-articular fractures developed stage-1 osteoarthritis, four patients $(15 \%)$ with intra-articular fractures developed stage-2 osteoarthritis, and three patients (33\%) with extra-articular fractures developed stage-1 osteoarthritis.

In the non operative treatment group, eleven patients $(48 \%)$ with intra-articular fractures developed stage- 1 osteoarthritis, six patients $(26 \%)$ with intra-articular fractures developed stage-2 osteoarthritis, and three patients (25\%) with extraarticular fractures developed stage-1 osteoarthritis. None of these patients with evidence of osteoarthritic changes reported any wrist pain.

Overall, there were significantly more complications in the operative treatment group than in the nonoperative treatment group (twelve compared with five) ( $p<0.05$; Fisher exact test). Twelve patients $(34 \%)$ from the operative treatment group had complications. No breakage of plates was observed. Five patients had extensor tenosynovitis because of screws that had penetrated the dorsal radial cortex. Four patients had development of flexor tenosynovitis which was aggravated by the position of the plate on the palmar rim of the distal radial surface, distal to the watershed line. The watershed line is defined as a transverse ridge that demarcates the distal aspect of the concave surface of the palmar aspect of the radius. Distal to this line, the radius slopes in a dorso-distal direction and becomes prominent palmarly. These patients were managed with implant removal. Carpal tunnel syndrome was observed in one patient. In that case, the carpal tunnel was released and the plate was removed. Two patients in the operative treatment group and five patients in the nonoperative treatment group had development of type-1 complex regional pain syndrome; in all patients, the symptoms resolved after treatment with physiotherapy and oral analgesics. No other complication was observed in the non-operative treatment group.

\section{Discussion}

According to the Gartland and Werley scoring system, twenty-two patients $(88 \%)$ had an excellent or good result and three patients $(12 \%)$ had a fair or poor result. One patient had development of complex regional pain syndrome (type 1).

Roumen et al. performed a prospective study of the treatment of distal radial fractures in patients older than fifty-five years ${ }^{[20]}$. All patients were managed initially with closed reduction and splinting. Two weeks after the reduction, $43 \%$ of the fractures had redisplaced (as indicated by a loss of $>10^{\circ}$ of dorsal angulation and $>5 \mathrm{~mm}$ of radial shortening). Half of these patients were managed with external fixation and the other half of these patients had continued nonoperative treatment. The patients who were managed with external fixation had better radiographic results, but did not have better functional results, in comparison with the patients who were managed nonoperatively. 
In a randomized controlled study by Bartl et al., 149 patients, who were $\geq 65$ years of age, were allocated to either volar locking plates or nonoperative treatment ${ }^{[10]}$. Patients with fracture re-displacement crossed over to plate fixation if it was discovered during study follow-ups, which resulted in $42 \%$ of patients in the nonoperative group actually being treated surgically. In our study, there were no plate fixations in the nonoperative treatment group. Our opinion is therefore that the results from the nonoperative treatment group in our study represent a realistic outcome after a completed nonoperative treatment of dorsally displaced distal radius fractures in an elderly population.

Egol et al. retrospectively compared the outcomes for patients over the age of sixty-five years in whom displaced distal radial fractures were treated operatively with plate fixation or external fixation (fortyfour patients) or nonoperatively with cast immobilization (forty-six patients) ${ }^{[21]}$. At twenty-four weeks, patients in the operative treatment group had better wrist extension than those in the nonoperative treatment group. At one year, this difference was not seen. There was no difference between the groups in terms of the DASH or pain scores at any time during the follow-up period. Grip strength was significantly better in the operative treatment group at one year. The radiographic results were better for the operative treatment group at each follow-up visit. There was no difference between the two groups with regard to complications. The authors concluded that minor limitations in the range of wrist motion and diminished grip strength, as seen with nonoperative treatment, did not limit function at one year. In the present study, ORIF with use of a volar fixedangle plate was associated with better radiographic results in comparison plaster immobilization for the treatment of distal radial fractures. There were no malunions and there was only a minor loss of reduction in the ORIF group. At the time of the latest follow-up examination, malunion occurred in all fractures in the nonoperative treatment group. Palmar tilt, radial inclination, and ulnar variance were significantly better in the ORIF group at the time of the latest follow-up examination.

There was a loss to follow-up of $13 \%$ in our study. It is a challenge to study fracture outcome in elderly patients because, comorbidities clearly diminish patients' ability to take part in clinical studies. Many patients declined to participate because of other illnesses.

Another aspect of difficulties with regard to clinical studies of elderly patients includes the analysis of patient-reported outcomes. To the best of our knowledge, it is largely unknown how the reliability of patient-reported outcome measure results may differ between young and elderly study participants. Neither the PRWE nor the DASH questionnaire has been validated in elderly patient populations. In the absence of other validated outcome measures, we still consider the PRWE and the DASH questionnaire to be the best available patient rated outcome measures for distal radial fractures in the elderly population.

Although there were no significant differences between the two treatment groups in terms of the mean active range of motion at the time of the latest follow-up examination, patients in the operative treatment group had better grip strength through the entire time period. The final PRWE and DASH outcome scores were not significantly different between the two groups. This finding is similar to those described by other authors who have reported on the treatment of distal radial fractures in the elderly ${ }^{[6,22]}$. The lack of association between the radiographic and functional outcomes among the elderly could be explained by a decreased demand on the upper extremity

associated with aging. It is questionable if a difference of $3 \mathrm{~kg}$ in grip strength is clinically important in this patient population ${ }^{[5]}$.

In the present study, the prevalence of post-traumatic osteoarthritis in patients with intra-articular fractures was significantly higher in the nonoperative treatment group than in the operative treatment group $(p<0.05 ; \mathrm{t}$ test for independent samples). This finding is similar to those of Knirk and Jupiter ${ }^{[18]}$, who reported a radiographic prevalence of $65 \%$ in patients with intra-articular distal radial fractures who were followed for a mean of 6.7 years.

The limited one-year follow-up period in our study may have been too short to determine if the radiographic signs of osteoarthritis would be symptomatic. The presence of osteoarthritis, however, is not always associated with pain or functional disability ${ }^{[22]}$. At the time of the latest follow-up examination, thirty-nine $(56 \%)$ of the seventy patients in our series had radiographic signs of arthritis, but none of them had any symptoms.

Overall, twelve patients $(34 \%)$ in the operative treatment group and five patients $(14 \%)$ in the nonoperative treatment group had complications. In total, ten patients $(29 \%)$ in the operative treatment group had secondary surgical procedures. No patient in the nonoperative treatment group required surgery.

Volar fixed-angle plate systems have made plate osteosynthesis popular for elderly individuals with osteoporotic bones. However, at twelve months after surgery, the active range of motion, the pain level, and the PRWE and the DASH scores were not different between the operative and nonoperative treatment groups. Although patients in the operative treatment group had better grip strength through the entire time period, achieving an almost anatomical reconstruction did not convey any improved range of motion or better ability of daily living activities in our cohort.

\section{Conclusion}

At the twelve-month follow-up, the range of motion, the level of pain, and the PRWE and DASH scores were not different between the operative and nonoperative treatment groups. Achieving anatomical reconstruction did not convey any improvement in terms of the range of motion or the ability to perform daily living activities.

\section{References}

1. Court-Brown CM, Caesar B. Epidemiology of adult fractures: a review. Injury. 2006; 37(8):691-7. Epub 2006 Jun 30

2. McQueen MM, Hajducka C, Court-Brown CM. Redisplaced unstable fractures of the distal radius: a prospective randomised comparison of four methods of treatment. J Bone Joint Surg Br. 1996; 78:404-9.

3. Martinez-Mendez D, Lizaur-Utrilla A, de-Juan-Herrero J. Intra-articular distal radius fractures in elderly patients: a randomized prospective study of casting versus volar plating. L Hand Surg Eur. 2018; 43(2):142-7. Epub 2017 Sep 4

4. Beumer A, McQueen MM. Fractures of the distal radius in low-demand elderly patients: closed reduction of no value in 53 of 60 wrists. Acta Orthop Scand. 2003; 74:98-100.

5. Young BT, Rayan GM. Outcome following nonoperative treatment of displaced distal radius fractures in low- 
demand patients older than 60 years. J Hand Surg Am. 2000; 25:19-28.

6. Barton T, Chambers C, Bannister G. A comparison between subjective outcome score and moderate radial shortening following a fractured distal radius in patients of mean age 69 years. J Hand Surg Eur. 2007; 32:165-9.

7. Tsukazaki T, Takagi K, Iwasaki K. Poor correlation between functional results and radiographic findings in Colles' fracture. J Hand Surg Br. 1993; 18:588-91.

8. Chung KC, Shauver MJ, Birkmeyer JD. Trends in the United States in the treatment of distal radial fractures in the elderly. L Bone Joint Surg Am. 2009; 91(8):1868-73

9. Arora R, Lutz M, Deml C, Krappinger D, Haug L, Gabi M. A prospective randomized trial comparing nonoperative treatment with volar locking plate fixation for displaced and unstable distal radius fractures in patients sixty-five years of age and older. J Bone Joint Surg Am. 2011; 93(23):2146-53

10. Bartl C, Stengel D, Bruckner T, Gebhard F; ORCHID Study Group. The treatment of displaced intra-articular distal radius fractures in elderly patients. Dtsch Arztebl Int. 2014; 111(46):779-87

11. Diaz-Garcia RJ, Oda T, Shauver MJ, Chung KC. A systemic review of outcomes and complications of treating unstable distal radius fractures in elderly. $\mathrm{J}$ Hand Surg Am. 2011; 36(5):824-35e2.

12. Ju JH, Jin GZ, Li GX, Hu HY, Hou RX. Comparison of treatment outcomes between nonsurgical and surgical treatment of distal radius fracture in elderly: a systematic review and meta-analysis. Langenbecks Arch Surg. 2015; 400(7):767-79. Epub 2015 Aug 30.

13. Chen Y, Chen X, Li Z, Yan H, Zhou F, Gao W. Safety and efficacy of operative versus nonsurgical management of distal radius fractures in elderly patients: a systematic review and meta-analysis. J Hand Surg Am. 2016; 41(3):404-13. Epub 2016 Jan 20.

14. MacDermid JC, Richards RS, Donner A, Bellamy N, Roth JH. Responsiveness of the short form-36, disability of the arm, shoulder, and hand questionnaire, patientrated wrist evaluation, and physical impairment measurements in evaluating recovery after a distal radius fracture. J Hand Surg Am. 2000; 25:330-40.

15. MacDermid JC. Development of a scale for patient rating of wrist pain and disability. J Hand Ther. 1996; 9:178-83.

16. McQueen MM, MacLaren A, Chalmers J. The value of remanipulating Colles' fractures. J Bone Joint Surg Br. 1986; 68:232-3.

17. Doro C, Hayden RJ, Louis DS. Complex regional pain syndrome type I in the upper extremity. Clin Occup Environ Med. 2006; 5:445-54, x.

18. Knirk JL, Jupiter JB. Intraarticular fractures of the distal end of the radius in young adults. J Bone Joint Surg Am. 1986; 68:647-59.

19. Villar RN, Marsh D, Rushton N, Greatorex RA. Three years after Colles' fracture. A prospective review. J Bone Joint Surg Br. 1987; 69:635-8.

20. Roumen RM, Hesp WL, Bruggink ED. Unstable Colles' fractures in elderly patients. A randomised trial of external fixation for redisplacement. J Bone Joint Surg Br. 1991; 73:307-11.

21. Egol KA, Walsh M, RomoCardoso S, Dorsky S, Paksima N. Distal radial fractures in the elderly: operative compared with nonoperative treatment. J Bone Joint Surg Am. 2010; 92:1851-7.

22. Karnezis IA, Panagiotopoulos E, Tyllianakis M, Megas
P, Lambiris E. Correlation between radiological parameters and patient-rated wrist dysfunction following fractures of the distal radius. Injury. 2005; 36:1435-9. 